Results: Of 788 abstracts, we included in the review 45 studies of which 1 systematic literature reviews, 16 cross-sectional studies, 15 cohort studies, 12 observational studies and 1 case-series study, with a total of 4656 patients. The mean age was $33.5 \pm 5.4$ years, while the mean disease duration was $97.4 \pm 65.2$ months. Figure 1 illustrates the quality of the included studies. Definitions of fertility/premature ovarian failure (POF) adopted in the studies varied in terms of the number of months of amenorrhea considered. Most studies did not use a hormonally based definition of fertility. Clinical factors associated with the development of POF were older age at the time initiation of therapy and older age at the onset of SLE disease. Cyclophosphamide exposure (CYC) and its cumulative dose influenced gonadal function in SLE women, leading to amenorrhoea and ovarian failure, as reported in 19 studies. Mycophenolate, azathioprine, calcineurin inhibitors and steroids seem to be associated with a lower risk of ovarian failure compared to CYC. 3 studies demonstrated that POF was more frequent in patients treated with CYC not receiving gonadotropin-releasing hormone analogues $(\mathrm{GnRH})$ in comparison to those co-treated with $\mathrm{GnRH}$. 11 studies evaluated the impact of damage and disease activity on ovarian reserve in patients with SLE with conflicting evidence. Finally, 18 studies investigated exposure to hormonal and serological factors able to influence fertility outcomes; among others nor Anti-Müllerian Hormone, neither anti-corpus luteum antibodies were associated with POF.

Conclusion: The role of disease activity on fertility in SLE patients is contradictory. Regarding management factors associated with fertility in SLE women of childbearing age, the strongest evidence is about the treatment with CYC and its cumulative dose. Hormonal and serological factors did not impact on fertility outcome but might be used as a surrogate of fertility, especially after treatment with disease-specific drugs. REFERENCES:

[1] Study Quality Assessment Tools NIH. https://www.nhlbi.nih.gov/ health-topics/study-quality-assessment-tools.

[2] Andreoli L. et al. EULAR recommendations for women's health and the management of family planning, assisted reproduction, pregnancy and menopause in patients with systemic lupus erythematosus and/or antiphospholipid syndrome. Ann Rheum Dis. 2017; 76: 476-485

Figure 1 - Risk of bias graph: review authors' judgements about each risk of bias using NIH risk-of-bias tool. 1A: systematic literature review/meta-analysis; 1B: observational studies; $1 \mathrm{C}$ : case series; green box = "yes/low risk of bias"; yellow box = "not applicable/not reported"; red box = "no/potential risk of bias"
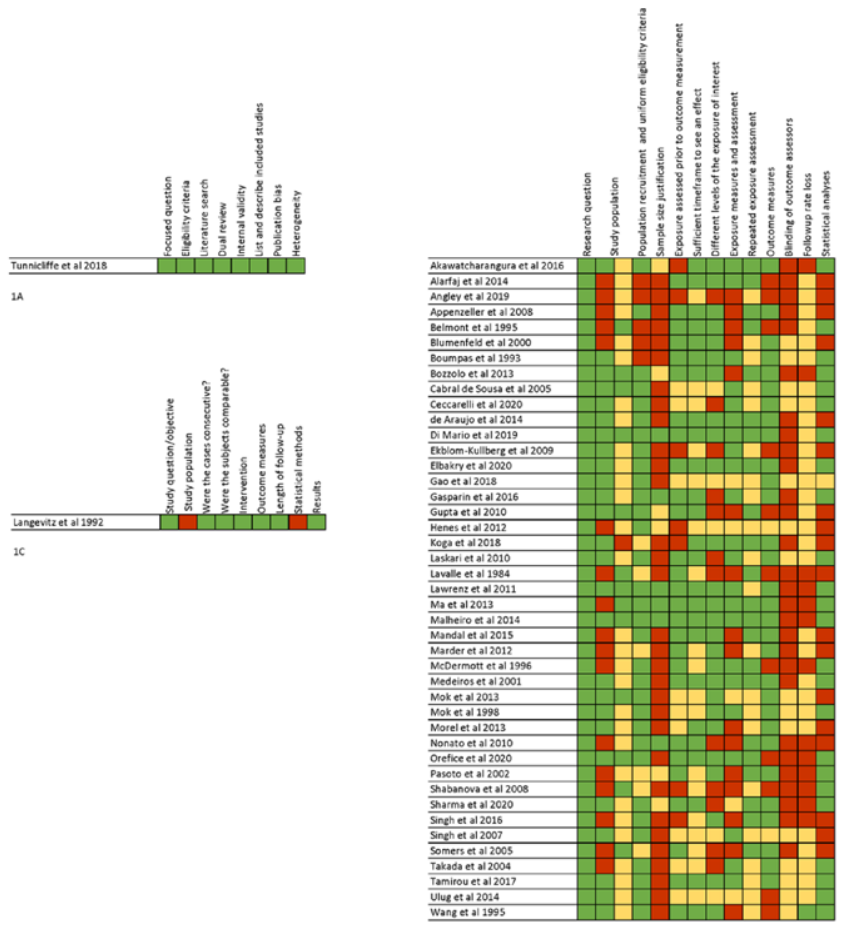

Disclosure of Interests: None declared

DOI: 10.1136/annrheumdis-2021-eular.937

\section{POS0717 AUTOIMMUNE LIVER DISEASE IN ANTICENTROMERE ANTIBODY POSITIVE PRIMARY SJOGREN'S SYNDROME}

B. Chalcev ${ }^{1}$, A. Torgashina ${ }^{1}$, E. Sokol ${ }^{1}$, J. Khvan ${ }^{1}$, V. Vasiliev ${ }^{2}$, S. Palshina ${ }^{3} .{ }^{1}$ V.A. Nasonova Research Institute of Rheumatology, Laboratory of Intensive Care of
Rheumatic Diseases, Moscow, Russian Federation; ${ }^{2}$ MEDSI, Department of Rheumatic Diseases, Moscow, Russian Federation; ${ }^{3}$ Clinic of Heart and Joint Diseases, Department of Rheumatic Diseases, Moscow, Russian Federation

Background: studies have shown that anticentromere antibody (ACA) positivity in primary Sjogren's syndrome (pSS) is associated with autoimmune liver diseases, most often primary biliary cholangitis (PBC) and autoimmune hepatitis $(\mathrm{AlH})[1,2,3]$, but detailed characteristics of the frequency and severity of liver disease in these patients is not presented in the literature.

Objectives: to identify the frequency, structure and characterize the course of autoimmune liver diseases in pSS+ACA.

Methods: we observe 82 patients with pSS+ACA. The diagnosis of pSS was established on the basis of Russian 2001 criteria, SSc was excluded based on the ACR/EULAR 2013 criteria [4]. 18 of 82 patients (22\%) had a persistent increase in alkaline phosphatase, 11 of them were positive for antimitochondrial antibodies (AMA) and, according to the recommendations of the American Association for the Study of Liver Diseases [5], they were diagnosed with PBC. 7 of 18 patients were AMA-negative, 2 of them had a liver biopsy and the diagnosis of AMA-negative PBC was confirmed, 4 patients who did not have a liver biopsy and 1 patient with hepatitis $B$ were excluded from the study. Also, in 6 of 64 patients without signs of liver damage, an increase in AMA was detected, in 1 of them a liver biopsy was performed and the diagnosis of PBC was confirmed. Thus, the group of patients with $\mathrm{pSS}+\mathrm{ACA}$ and autoimmune liver diseases included 19 patients: 12 patients with AMA-positive PBC, 2 patients with AMA-negative PBC and 5 patients with asymptomatic AMA positivity.

Results: The median follow-up for 19 patients with pSS+ACA and autoimmune liver diseases was 4 years. AMA were detected in $89.5 \%$ of patients, an increase in IgM - in 42.1\%, an increase in ALT / AST - 63.2\%, a decrease in albumin, prothrombin index and cytopenia - $15.8 \%$ (were associated with the development of liver cirrhosis). In most cases, the clinical course of liver disease was characterized by an asymptomatic, slowly progressing course, with no signs of progression during observation. Cirrhosis and portal hypertension were detected in $15.8 \%$ of patients, hepatic encephalopathy - in 10.5\%. Liver biopsy was performed in 9 patients, PBC was diagnosed in all cases (overlap syndrome with AlH was established in 3 cases). Assessment of PBC histological stages showed signs of stage 1 in 5 patients, stage 2 in 1 patient, stage 3 in 3 patients. Observation of 5 patients with stage $1 \mathrm{PBC}$ and 5 AMA-positive patients without signs of liver damage (median follow-up was 2 years), showed the absence of clinical, laboratory and instrumental progression of liver disease, which is why we believe that these patients have epithelitis of the biliary ducts as manifestation of glandular lesions in pSS, but not PBC.

Conclusion: autoimmune liver diseases in pSS+ACA are detected in $23.2 \%$ of patients, most of whom develop PBC and epitheliitis of the biliary ducts with the same frequency, less often overlap syndrome of $\mathrm{PBC}$ and $\mathrm{AlH}$, and characterized by a mild, slowly progressing course and rarely lead to liver cirrhosis.

\section{REFERENCES:}

[1] Masako Kita et al. Abnormal Liver Function in Patients with Sjogren's Syndrome. Acta Med. Nagasaki 41: 31-37.

[2] Baldini, Chiara et al. "Overlap of ACA-positive systemic sclerosis and Sjögren's syndrome: a distinct clinical entity with mild organ involvement but at high risk of lymphoma." Clinical and experimental rheumatology vol. 31,2 (2013): 272-80

[3] Bournia, Vasiliki-Kalliopi K et al. "Anticentromere antibody positive Sjögren's Syndrome: a retrospective descriptive analysis." Arthritis research \& therapy vol. 12,2 (2010): R47. doi:10.1186/ar2958.

[4] van den Hoogen, Frank et al. "2013 classification criteria for systemic sclerosis: an American College of Rheumatology/European League against Rheumatism collaborative initiative." Arthritis and rheumatism vol. 65,11 (2013): 2737-47. doi:10.1002/art.38098

[5] Lindor, Keith D et al. "Primary Biliary Cholangitis: 2018 Practice Guidance from the American Association for the Study of Liver Diseases." Hepatology (Baltimore, Md.) vol. 69,1 (2019): 394-419. doi:10.1002/hep.30145.

Disclosure of Interests: None declared

DOI: 10.1136/annrheumdis-2021-eular.949

\section{\begin{tabular}{|l|l}
\hline POS0718 CLINICAL CHARACTERISTICS ASSOCIATED \\
\hline
\end{tabular} WITH GLANDULAR INVOLVEMENT EVALUATED BY SALIVARY GLAND ULTRASONOGRAPHY IN SJÖGREN'S SYNDROME}

T. Shimizu $^{1}$, H. Nakamura ${ }^{1}$, Y. Takagi ${ }^{2}$, S. Y. Nishihata ${ }^{1}$, R. Sumiyoshi ${ }^{1}$, T. Igawa ${ }^{1}$, T. Koga ${ }^{1}$, S. Y. Kawashiri ${ }^{1}$, N. Iwamoto ${ }^{1}$, K. Ichinose ${ }^{1}$, M. Tamai ${ }^{1}$, T. Origuchi ${ }^{1}$, A. Kawakami ${ }^{1}{ }^{1}$ Nagasaki University Graduate School of Biomedical Sciences, Department of Immunology and Rheumatology, Nagasaki, Japan; ${ }^{2}$ Nagasaki University Graduate School of Biomedical Sciences, Department of Radiology and Cancer Biology, Nagasaki, Japan 
Background: Sjögren's syndrome (SS) is a systemic autoimmune disease characterized by periductal lymphocytic infiltration of the salivary and lacrimal glands, which results in reduced secretory functions and oral and ocular dryness (1). In addition, patients often have extraglandular manifestations, such as interstitial pneumonia and interstitial nephritis, and the appearance of autoantibodies such as anti-Ro/SS-A and La/SS-B antibodies. Salivary gland ultrasonography (SGUS) is typically used to evaluate the findings of salivary glands; thus, we can evaluate the severity of salivary gland disorders due to SS using SGUS in real time (2).

Objectives: To identify clinical indices, including disease activity, associated with glandular involvement evaluated using SGUS in patients with SS.

Methods: We enrolled patients with SS $(n=115)$ and non-SS sicca subjects $(n=90)$ who visited Nagasaki University Hospital between 1995 and 2019. The patients' SS classifications were based on the 2002 American-European Consensus Group (AECG) SS classification criteria (3). The non-SS sicca subjects exhibited sicca symptoms but did not fulfill the AECG SS classification criteria. SGUS and clinical indices such as age, sex, the focus score (FS), sicca symptoms, the Saxon test results, Schirmer's test results, anti-SS-A/Ro antibody positivity, anti-SS-B/La antibody positivity, anti-centromere antibody (ACA) positivity, serum immunoglobulin G levels, and the clinical European League Against Rheumatism SS disease activity index were examined. The ultrasonography (US) score was calculated based on SGUS imaging (hypoechoic area, hyperechoic band, and irregular border) (4).

Results: The US score was significantly higher in patients with SS than that in non-SS sicca subjects. In addition, we found significant correlations between the US score and FS in patients with SS. Multivariate analysis revealed the FS, Saxon test positivity, and ACA positivity as the variables independently associated with the US score in patients with SS. These results were the same in the primary SS patient group $(n=96)$. Patients with ACA positivity had significantly higher US scores compared to those in patients with ACA negativity, whereas the FS was not significantly high. In addition, patients with ACA positivity had significantly greater positivity of hyperechoic bands than that in patients with ACA negativity.

Conclusion: This study indicated that ACA positivity, which is not reflected in sialadenitis of SS, is associated with the US score in patients with SS. These results suggest that US findings of patients with ACA positivity might show specific changes in salivary glands, such as fibrosis, and not only sialadenitis (5).

REFERENCES:

[1] Ramos-Casals M, Tzioufas AG, Font J. Primary Sjogren's syndrome: new clinical and therapeutic concepts. Ann Rheum Dis. 2005;64(3):347-54.

[2] van Ginkel MS, Glaudemans A, van der Vegt B, Mossel E, Kroese FGM, Bootsma $\mathrm{H}$, et al. Imaging in Primary Sjogren's Syndrome. J Clin Med. 2020;9(8).

[3] Vitali C, Bombardieri S, Jonsson R, Moutsopoulos HM, Alexander EL, Carsons SE, et al. Classification criteria for Sjogren's syndrome: a revised version of the European criteria proposed by the American-European Consensus Group. Ann Rheum Dis. 2002;61(6):554-8.

[4] Takagi Y, Nakamura H, Sumi M, Shimizu T, Hirai Y, Horai Y, et al. Combined classification system based on ACR/EULAR and ultrasonographic scores for improving the diagnosis of Sjogren's syndrome. PLoS One. 2018;13(4):e0195113.

[5] Nakamura H, Kawakami A, Hayashi T, Iwamoto N, Okada A, Tamai M, et al. Anti-centromere antibody-seropositive Sjögren's syndrome differs from conventional subgroup in clinical and pathological study. BMC Musculoskelet Disord. 2010;11:140.

Disclosure of Interests: None declared

DOI: 10.1136/annrheumdis-2021-eular.1110

\section{POS0719 ADVERSE OUTCOMES AND REHOSPITALIZATION AFTER DELIVERY AMONG WOMEN WITH SYSTEMIC LUPUS ERYTHEMATOSUS OR RHEUMATOID ARTHRITIS AND THEIR INFANTS}

J. Sabo ${ }^{1}$, N. Singh ${ }^{1}$, D. A. Crane ${ }^{2}$, D. R. Doody ${ }^{3}$, M. A. Schiff ${ }^{4,5,6}$, B. A. Mueller ${ }^{3,6}$ ${ }^{1}$ University of Washington, Division of Rheumatology, Seattle, United States of America; ${ }^{2}$ University of Washington, Department of Rehabilitation Medicine, Seattle, United States of America; ${ }^{3}$ Fred Hutchinson Cancer Research Center, Public Health Sciences Division, Seattle, United States of America; ${ }^{4}$ University of Washington, Department of Obstetrics and Gynecology, Seattle, United States of America; ${ }^{5}$ University of New Mexico, Albuquerque, Division of Epidemiology, Biostatistics \& Preventive Medicine, Albuquerque, United States of America; ${ }^{6}$ University of Washington School of Public Health, Department of Epidemiology, Seattle, United States of America

Background: Women with rheumatoid arthritis (RA) or systemic lupus erythematosus (SLE) have greater risk of adverse obstetric and birth outcomes than women without these conditions. Infant outcomes are less well-studied. It is unknown whether re-hospitalization after delivery occurs more often for affected mothers and their infants.

Objectives: We compared obstetric outcomes among women with and without RA or SLE, and birth outcomes among their infants. Maternal and infant rehospitalizations $<2$ years of delivery were also compared.

Methods: This population-based cohort study used linked birth-hospital discharge data from Washington State for 1987-2014. International Classification of Disease $9^{\text {th }}$ revision (ICD9) codes identified all women with RA (ICD9 714.X, 725.X) and SLE (ICD9 710, 710.0, 710.1) in the hospital discharge record at delivery, and a 10:1 comparison group of women without these codes. Analyses were restricted to singleton live births (1,223 RA; 1,354 SLE). Poisson regression with robust standard errors estimated relative risks (RR) and 95\% confidence intervals $(\mathrm{Cl})$ for selected outcomes, accounting for delivery year, maternal age, and parity.

Results: Many adverse outcomes were more common among RA and SLE cases than among comparison women. Preeclampsia occurred more often during pregnancies of women with RA (RR 1.42, 95\% $\mathrm{Cl} 1.17-1.71$ ) or SLE (RR 2.33, 95\% Cl 2.01-2.70), as did preterm rupture of membranes (PROM, RR $2.85,95 \% \mathrm{Cl} 2.20-3.72$ for RA; RR $3.28,95 \% \mathrm{Cl} 2.54-4.23$ for SLE) Cesarean deliveries were more common among nulliparous women in both groups (RR 1.32, 95\% Cl 1.18-1.48 for both conditions). Infants of women with RA or SLE were more likely to weigh $<2500 \mathrm{~g}$ (RR 2.08, 95\% Cl 1.72-2.52 for RA; RR 4.88, 95\% Cl 4.27-5.58 for SLE), be small for gestational age (RR $1.25,95 \% \mathrm{Cl} 1.07-2.50 ; \mathrm{RR} 2.30 ; 2.04-2.59$, respectively), delivered at $<32$ weeks gestation (RR 1.83, 95\% Cl 1.13-2.97; RR 5.13, 95\% Cl 3.75-7.01 respectively), and require neonatal intensive care unit admission (NICU, RR 1.89, 95\% Cl 1.56-2.30; RR 2.71, 95\% Cl 2.25-3.28, respectively). Infants of women with SLE were more likely to have a malformation (RR $1.46,95 \% \mathrm{Cl}$ 1.21-1.75) or die within 2 years (RR 2.11, 95\% Cl 1.21-3.67). Rehospitalization levels among both women with RA (RR 2.22; 1.62-3.04) and SLE (RR 2.78 $95 \% \mathrm{Cl} 2.15-3.59)$ were greatest $<6$ months of delivery and declined over time. Infants of women with SLE had increased rehospitalization $<6$ months (RR 1.64, 95\% Cl 1.36-1.98).

Conclusion: Consistent with prior literature, we found women with RA or SLE experienced many adverse outcomes. In our data, these included preeclampsia, PROM, and cesarean deliveries, with increased risks more notable among women with SLE. Infants of women with either condition were more likely to weigh $<2500 \mathrm{~g}$, be $<32$ weeks gestation, small for gestational age, and require NICU admission than infants of comparison women. Only infants of women with SLE had increased malformations. Maternal rehospitalization after delivery was more common in both groups; most marked at $<6$ months. Infant rehospitalizations were increased in both cohorts to a lesser extent. Close follow-up during this time period is crucial to minimize adverse outcomes.

Disclosure of Interests: Julianna Sabo: None declared, Namrata Singh: None declared, Deborah A. Crane: None declared, David R. Doody: None declared, Melissa A. Schiff: None declared, Beth A. Mueller Shareholder of: Household owns shares in AstraZeneca

DOI: 10.1136/annrheumdis-2021-eular.1127

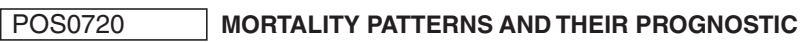 VALUE IN PATIENTS WITH SYSTEMIC LUPUS ERYTHEMATOSUS IN CUBA}

H. Hernández-Negrín ${ }^{1}$, Y. Roque-Dapresa ${ }^{2} .{ }^{1}$ Universidad de Ciencias Médicas de Villa Clara, Internal Medicine, Santa Clara, Cuba; ${ }^{2}$ Hospital Universitario Arnaldo Milián, Internal Medicine, Santa Clara, Cuba

Background: There is an increase over time in studies adopting cluster analysis (CA) to identify causes, patterns and severity in rheumatic diseases. ${ }^{1}$ However $\mathrm{CA}$ has not been used to identify patterns of causes-of-death in patients with systemic lupus erythematosus (SLE)

Objectives: 1) to identify homogeneous groups of patients according to their causes-of-death, 2) to identify homogeneous groups of cause-of-death combinations and 3) to determine the potential prognostic value of the identified patterns. Methods: This is a population-based study using the mortality register of Villa Clara (province of Cuba) between 1990 and 2019. Based on International Classification of Disease (ICD), Nine and Tenth Revision (ICD-9, code 710.0 and ICD-10, code M32), there were identified the SLE-related deaths (any mention on the death certificate) and their associated causes-of-death. Two hierarchical CA with Ward's method was carried out, based on the associated causes-ofdeath: one to conglomerate patients and another one to conglomerate variables. To identify differences in socio-demographic characteristics and causes-of-death between each cluster, Chi-square test, Fisher's exact test and Kruskal-Wallis tes were used as appropriate. Multivariate Poisson regression model corrected for over-dispersion was used to determine the prognostic value of the clusters. Years of potentially life lost (YPLL) was defined as a dependent variable. To calculate 